\title{
Malaria: The Dilemmas of Malarial Diagnostics
}

\author{
MW Johnson, $\mathrm{N}$ Ineson
}

\section{Introduction}

In clinical practice the diagnosis of malaria is usually relatively easy. In the acute situation a blood film is sent and if the result is positive the patient is started on appropriate therapy. The dilemma arises if after several days a patient still exhibits symptoms and signs of malaria yet numerous blood films for parasites have not provided a conclusive diagnosis. Is there a place for blind therapy?

This article looks at the guidelines set out by the School of Hygiene and Tropical Medicine and demonstrates that the criteria for diagnosis, tracking improvement and discharge, although straightforward, are not always easy to implement. The difficulties with traditional diagnosis are examined with regard to a cohort of 21 patients admitted to Frimley Park Hospital over a three-month period. All patients had a diagnosis of pyrexia thought to be malaria, having recently returned from an area with endemic malaria. We will also consider the effectiveness and cost efficiency of alternative investigations for malaria.

\section{Prevalence}

Malaria is caused by the plasmodium species of which there are four types: P.falciparum, P.malariae, P.vivax and P.ovale. Plasmodium falciparum is still a major worldwide cause of death. Together these parasitic species affect between 300-500 million people and kill 1-3 million people annually. Ninety percent of these malarial mortalities occur in subSaharan Africa (1).

\section{Symptoms and Signs}

Fever and rigors are almost universal in cases of malaria. Other symptoms include malaise, myalgia, headache and haematuria. Clinical signs include a raised temperature, splenomegaly, hepatomegaly and jaundice. Common laboratory findings include anaemia and mild elevations of serum bilirubin. White blood cell counts tend to be normal or decreased, but can be raised (2).

MW Johnson

BSc MRCP (UK)

Bromley NHS Trust, Bromley, Kent, BR2 9AJ

Col $\mathrm{N}$ Ineson FRCP L/RAMC

MDHU Frimley Park Hospital, Frimley, Surrey, GU11 5UJ

\section{Malaria Prevention}

The most effective prevention of malaria is avoidance of mosquito bites. For many people (especially soldiers who spend much time in outdoor exercises) this can prove exceptionally difficult. Mosquito nets impregnated with insecticides, the use of personal insecticides, and even simple measures such as keeping sleeves rolled down have been found to be effective. It is also advisable to use nocturnal air conditioning, which is an impossibility for many budget travellers and soldiers on operations (3). Not forgetting these simple precautions travellers to endemic areas should take appropriate chemoprophylaxis. Chloroquine is active against P.ovale, P.malariae and most P.vivax, however chloroquine resistant P.falciparum is prevalent. Proguanil has little effect against the benign malarial species and proguanil resistant P.falciparum is becoming increasingly common. The combination of both drugs is more effective than either one alone (4). Mefloquine has been shown to be highly efficacious especially against P.falciparum and this is why it was used as the drug of choice given to the soldiers prior to them being dispatched to Sierra Leone. These drugs are started 3 week before leaving for an endemic region. This is not to build up intravascular drug levels, but to ensure there are no adverse reactions and side effects. Over recent years the medical profession has become increasingly aware of the widespread resistance of P.falciparum to the above drugs and more recently to pyrimethamine and sulphadoxine (Fansidar). Chemoprophylaxis cannot be seen as a guarantee of immunity. This is compounded by widespread public concern about potential side effects of antimalarials, which may reduce compliance. Despite using the best available prophylaxis in malarial environments a proportion of travellers will inevitably develop the disease.

\section{Diagnosis}

The finding of parasites in a blood film usually produces a diagnosis. In areas where malaria is endemic adults are often found to have developed semi-immunity with a significant parasitaemia of $50+$ malaria parasites per 200 blood cells. This is not the case with Caucasian travellers returning from these endemic areas who have contracted malaria. In these patients a single parasite on a blood film represents a positive diagnosis of malarial illness.

\section{Investigation}

The standard method of investigation in most medical units worldwide is still microscopic examination of blood films. Newer methods are now being looked at such as immunochromatographic detection of circulating parasite antigen and poly- 
merase chain reaction testing for plasmodium antigen.

It is said that if a person has malaria a positive diagnosis can be made from the blood films $95 \%$ of the time. In hyperendemic areas where laboratory staff are experienced in malarial diagnostics such as on the Thai-Myanmar border, their sensitivities reach $94 \%$ with a specificity of $95 \%$, a positive predictive value of $95 \%$ and a negative predictive value of $96 \%$. However a high level of input from the international laboratory technician performing training, follow-up and evaluation was required (5).

Although the gold standard diagnostic test for malaria is light microscopy of giemsa stained thick and thin films, this method is time consuming and its preparation can take up to 60 minutes. Ideally blood should be used from a finger prick however if prepared quickly blood obtained from venepuncture and collected in heparin- or EDTA-coated tubes is acceptable (6). The thick smear makes the diagnosis and provides the sensitivity of the technique, whilst the thin smear evaluates the parasitaemic count and thus gives the test its specificity. However, inexperienced parasitologists and on-call laboratory staff may miss them. Aspects of storage in relation to time, type of anticoagulant and subsequent interpretation certainly need careful consideration before making a diagnosis (7). Acridine orange staining using an interference filter in a light microscope has been shown to be four times more sensitive than the conventional giemsa stain (8). It can show positivity with much lower parasite counts as compared with other stains. Traditional diagnosis using giemsa stain thick and thin blood smears is inappropriate for many areas, as there are insufficient microscopes and/or trained microscopists to read and interpret the slides.

Although PCR based methods are arguably the most sensitive (9), they are also the most costly, requiring specialised equipment and specialty training. It is thus unlikely to be useful in the routine diagnosis of malaria. This technique tends to be used in specialist centres and is particularly useful when studying different strains, mutations and genes involved in drug resistance (10).

The diagnosis of malaria worldwide is often made purely on the basis of clinical symptoms. In 1996 the WHO claimed that at best this would be approximately 50\% accurate. It is argued that fever relates directly to parasite density. However evidence from several groups including papers by Eintertz and Bates suggested that there was a poor association between significant parasitaemic counts and reported or documented fever. They go on to say "....in the absence of fever on examination, history of fever should be supported by clinical signs such as splenomegaly, anaemia, or jaundice before antimalarial treatment is prescribed." (11).

\section{Audit}

During the 3 months between 24/05/00 and the $31 / 08 / 00$ we observed a total of 21 male patients who were admitted to Frimley Park Hospital after returning from the tropics. The medical notes and laboratory investigations were reviewed to assess symptoms, diagnosis, number and type of diagnostic investigations, eradication review and length of stay. In all 21 cases the above information was acquired.

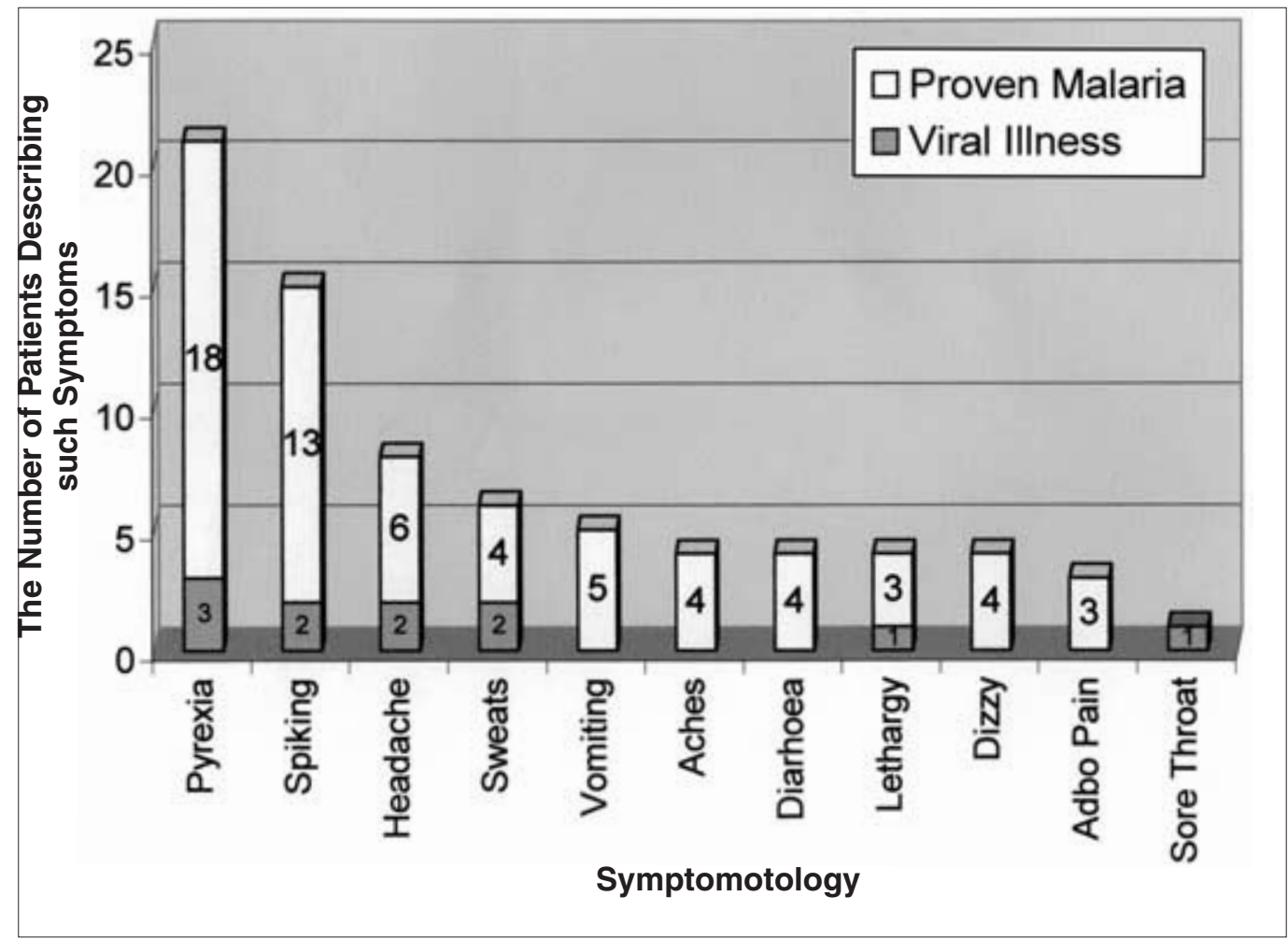




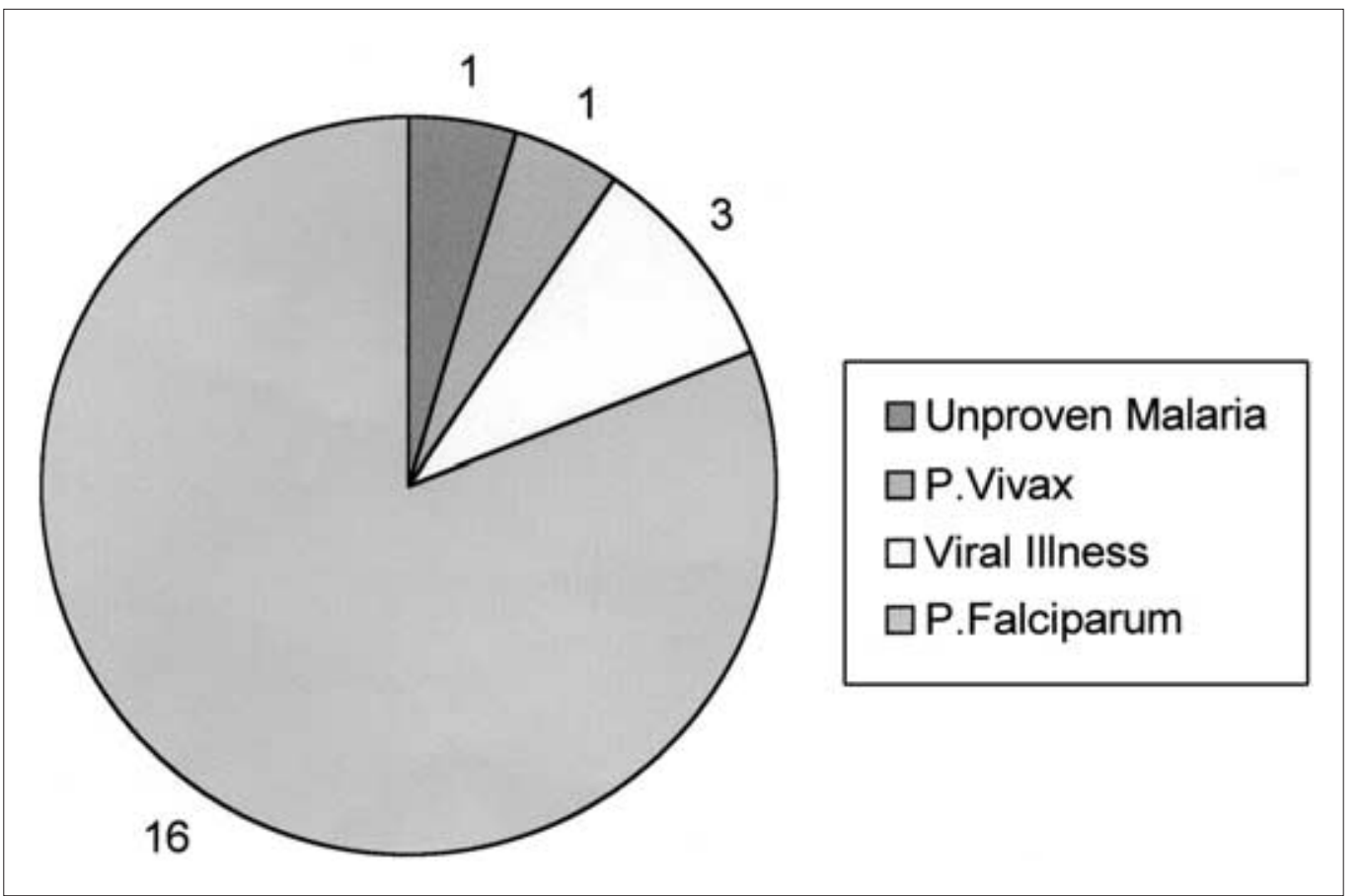

The final diagnosis in the 21 men presenting with pyrexias presumed to be Malaria after returning from the Tropics.

The aim of the audit was firstly to assess the speed with which a positive diagnosis was made and appropriate treatment started, and secondly to ensure that a rapid but safe discharge was being made.

All 21 patients had a pyrexial illness thought to be malaria. Seventeen of these patients were army personnel, 16 of whom had returned from Sierra Leone. All of the patients involved in this study had taken malarial prophylaxis, although clearly there may have been problems with compliance. Some soldiers deployed to Sierra Leone started taking mefloquine later than others. Chemoprophylaxis was issued in the form of mefloquine, however one patient had taken chloroquine and proguanil. All 21 patients complained of fever, 18 of which were confirmed on admission with temperatures ranging from 37.2 to $40.2^{\circ} \mathrm{C}$ of which 15 were shown to have the classical pattern of spiking pyrexia. A variety of other symptoms were reported and are shown in the Figures. Of the 21 patients, 17 had proven malaria, 16 of which were in the form of P.falciparum and 1 of which was shown to be P.vivax. One patient was treated empirically for malaria although no positive diagnosis was ever made. It was felt by the medical team looking after this patient that the symptoms, abnormalities in liver function and clinical findings of hepatosplenomegaly were in keeping with the diagnosis of malaria. He dramatically improved with the start of antimalarial medication. The other 3 patients were diagnosed as having viral illnesses.

Twelve of the patients were diagnosed on the initial blood film. Five of the malarial cases required 2 or more films over the course of a 3-day period to make the diagnosis. In one of these difficult diagnostic cases an ICT test gave a clear indication of the presence of malarial parasites on the first blood sample sent, however it was not until the third blood film that malarial parasites were actually seen. The medical team looking after this patient used this test as a clear indication to start anti-malarial medication early. In one other patient that proved difficult to diagnose, a positive report was obtained from samples sent to the School of Hygiene and Tropical Medicine. All the patients diag-nosed with P.falciparum were treated with oral quinine with the exception of two that required their medication intravenously due to profuse vomiting and high pyrexias. Interestingly the average length of stay in hospital was markedly reduced for the intravenous quinine users which was 4 days as compared to 5.2 in those taking oral quinine. The patient with P.vivax was treated with a course of oral chloroquine followed by primaquine.

Prior to discharge the patients had been asymptomatic for an average of 2.5 days (ranging from 1 to 6 ) and had at least 1 to 3 clear films. Their length of stay in hospital was 4.3 days on average (varying from 1 to 8 days).

\section{Conclusion}

At present the School of Hygiene and Tropical Medicine advise taking 3 blood films a day until the diagnosis is made. Should there be any contention in making the diagnosis they offer a review service with PCR testing if necessary. The use of antimalarials should only be used in those with definite evidence of malarial parasites on blood films. They do not advocate the use of empirical treatment. P.falciparum should be treated with IV quinine if the patient is highly 
symptomatic, has a parasite count $>2 \%$, or has a pyrexia $>39^{\circ} \mathrm{C}$. If this is not the case oral quinine can be used and continued for a minimum of 3 days and a maximum of 7 days after negative blood films. A once only dose of Fansidar (3 tablets) should then be given in an attempt to clear any latent hypnozoites within the liver. A safe discharge can be made when the patient is symptomatically well with at least 1 clear blood film. Patients with benign malaria tend not to need hospital admission unless highly symptomatic. They can be treated as outpatients with oral chloroquine. It is with the above advice from the School of Hygiene and Tropical Medicine that we compared our own practice.

From our audit one can see that despite chemoprophylaxis individuals still develop malaria. Most patients were diagnosed on their initial blood films. Some of the patients were highly symptomatic with very low parasite counts, which made it difficult to acquire a quick diagnosis from the blood films. These patients required more than one blood smear to make a diagnosis, and this in some cases took several days. It was unusual to see more than one blood film a day being carried out on each patient and this could have led to further delays in diagnosis. Flooding the haematology department with blood films however, may have become counter-productive. A quicker, simpler and more sensitive method of detecting malaria would obviously be beneficial. Such a test could reduce hospital length of stay by reaching a diagnosis more quickly and allowing treatment to commence as soon as possible. Despite the clear guidelines set out by the School of Hygiene and Tropical Medicine only 2 of the 5 patients that reached criteria for intravenous quinine actually received it. It was, therefore, interesting to see that there was a significantly shorter length of stay in those receiving intravenous as compared to oral quinine.

Many of the men on medication had been asymptomatic for several days with at least 1 to 3 clear blood films prior to discharge. Clear-cut guidelines of the criteria necessary to make an early but safe discharge would also be helpful. Patients need to be symptomatically fit for discharge but given the difficulties of demonstrating positive blood films in some cases, is one negative film enough to convincingly claim eradication of intravascular malarial parasites? It is not uncommon in P.falciparum malaria for the parasites to be sequestered and they are not always detectable in the peripheral blood circulation. This probably plays an important part in the number of false negatives seen in this diagnostic procedure (10). Some doctors prefer to see at least three clear blood films before being confident on a safe discharge. This practice is obviously safer, but costly, as it significantly increases the length of stay in hospital.

\section{Discussion}

One of the most promising breakthroughs in malarial diagnostics are the serological dipstick tests, for example: ParaSightF test (Becton Dickinson, USA) and ICT Malarial Pf test (ICT, Australia). These are antigen capture assays based on detecting histidine rich protein 2 (HRP - 2), a water-soluble antigen expressed by Plasmodium falciparum trophozoites. Their accuracy is comparable to each other, and not dissimilar to PCR (12). They are simple to use, easy to interpret and produce results within 15 minutes. The ICT Malarial Pf test is said to be the easiest to use and gives results within 5 minutes. It has a sensitivity of $96 \%$, a specificity of $98 \%$, a positive predictive value of $98 \%$ and a negative predictive value of $96 \%$ (13). These serological tests are felt to be almost as sensitive as microscopic examination carried out by experts. However there is only a $50-70 \%$ sensitivity in samples with less than 50 parasites per microlitre $(\mathrm{mcl})$. In endemic areas this is unlikely to be a problem as treatment is rarely started in those with a parasite count less than 500 . However, it may cause a problem in travellers from non-endemic regions. It is unlikely that patients would be misdiagnosed by the dipstick tests as the vast majority of patients have parasite counts greater than 50 per mcl. Their cost at present is still a limiting factor, costing approximately. \$12 US in Europe for two tests. However, one has to take into account the savings in technical time, preparation time and the cost of buying and maintaining expensive equipment. Microscopy has the advantage of covering all malarial species, allows an estimate on the parasitaemic count and can determine which growth stage the malarial parasites are in. The serological tests may be useful as a screen for febrile patients and although further blood film exam-inations are still required the benefits as a "side room" investigation or use in the field in remote areas are clear. It provides rapid accurate diagnosis in situations where high technology equipment is unavailable. There is no special training required and the test can also be used to distinguish between the benign malarias and the potentially lethal falciparum malaria. Studies have already assessed the effectiveness of these tests in the forest villages in the Mandla district of central India with impressive results (14).

In the audit carried out at Frimley Park Hospital the ICT test was used on 3 occasions in patients requiring 2 or more blood films. Their results were comparable with microscopy and in one of these difficult diagnostic cases the ICT test gave a clear indication of the presence of malarial parasites on the first blood sample sent, 
whilst a positive microscopic diagnosis was not made until the third blood film. This enabled the medical team involved to commence early antimalarial treatment and would have significantly reduced hospital admission time.

There is clearly a role for quicker and simpler malarial diagnostic tests that have a high sensitivity and specificity, both in the field and also in hospital medicine. The ability to make an early diagnosis and convincingly prove eradication would obviously have a major effect on hospital admission time. The population group studied here is dissimilar to those looked at in endemic regions. The fact that all of our patients took malarial prophylaxis sets them apart in the way that they presented and may explain the low parasitaemic counts and difficulty in making a quick positive diagnosis. In this day and age of prophylaxis for travellers and increasing drug resistance one wonders whether or not we are selecting out highly virulent strains. Traditional views on malarial management may have to change in accordance with the changing pattern of the disease that we are now seeing in this country.

\section{Acknowledgements}

I would like to acknowledge Lt. Col Hoad and Col Fabricius for their support, and for allowing us to use their patients in this study.

\section{References:}

1. White NJ, Nosten F, Looareesuwan S Watkins WM, Marsh K, Snow RW, et al. Averting a malarial disaster. Lancet 1999; 353: $1965-7$.

2. Bradley DJ, Warhurst DC. Guidelines for the prevention of malaria in travelers from United Kingdom Commun Dis Rep. CDR Rev 7:R137-52
19971997.

3. Tham JM, Lee SH, Tan TM, Ting RC, Kara UA. Detection and species determination of malarial parasites by PCR: comparison with microscopy and with ParaSight-F and ICT malaria PF tests in a clinical environment. Fournal of Clinical Microbiology. May 1999; 37(5): 1269-73.

4. Warhurst DC, Williams JE. Laboratory diagnosis of malaria. Fournal of Clinical Pathology. 1996; 49: 794796.

5. Baudon D. Malaria and travellers - practical aspects. Tropical medicine and parasitology. Sep 1993; 44(3): 246-9.

6. Makler MT, Palmer CJ, Ager AL. A review of practical techniques for the diagnosis of malaria. Annals of Tropical Medicine and Parasitology. Vol 92, 1998, 4: 419 - 433 .

7. Kortepeter M, Brown J. A review of 79 patients with with malaria seen at a military hospital in Hawaii from 1979 to 1995. Military Medicine. Feb 1998; 163:84-89.

8. Hemme F, Gay F. Internal quality control of the malarial microscopy diagnosis for 10 laboratories on the Thai-Myanmar border. Southeast Asian fournal of Tropical Medicine and Public Health Sept 1998; 29(3):529-36.

9. Einterz EM, BatesME. Fever in Africa: do patients know when they are hot? Lancet 1997; 350: 781.

10. Warhurst DC, Williams JE. Laboratory diagnosis of malaria. F Clin Pathol 1996; 49: 533-8.

11. Agbani Hind MM, El Has Imad Abdain, El Town Islam Abdalla, El Hassan AM. Fluorescence microscopy using light microscope filled with an interference filter for the diagnosis of malaria. Trans Roy Soc Trop Med Hyg. 1994; 88: 61 - 4 .

12. Pierani P, Mills C D, Ohrt C, Harrington M A, Kain KC. Comparison of the ParaSightF test and the ICT Malarial PF test with the polymerase chain reaction for diagnosis of Plasmodium Falciparum in travellers. Trans $R$ Soc Trop Med Hyg 1998 MarApril; 92 (2): 166-9.

13. Cavallo JD, Fernandez E, Gerome P, Platten N, Debord T, Le Vogueresse R. Serum HRP-2 Ag and imported PF malaria: Comparison of ParaSightF and ICT malaria PF. Med Trap (Mars) 1997; 57(4): 353-6.

14. Singh N, Volecha N, Sharma VP. Malaria diagnosis by field workers using and immunochromatograhic test. Trans R Soc Trap Med Hyg 1997 July-Aug; 91(4): 396-7. 